\title{
Les enjeux de la professionnalisation des entreprises
}

d'économie sociale

The issues of professionalization for social economy

enterprises

\section{Los desafíos de la profesionalización de las empresas de economía social}

\section{Luc Audebrand et Myriam Michaud}

Numéro 338, octobre 2015

ESS et mutations du travail et de l'emploi

URI : https://id.erudit.org/iderudit/1033873ar

DOI : https://doi.org/10.7202/1033873ar

Aller au sommaire du numéro

Éditeur(s)

Association Recma

ISSN

1626-1682 (imprimé)

2261-2599 (numérique)

Découvrir la revue

Citer cet article

Audebrand, L. \& Michaud, M. (2015). Les enjeux de la professionnalisation des entreprises d'économie sociale. Revue internationale de l'économie sociale,

(338), 54-68. https://doi.org/10.7202/1033873ar
Résumé de l'article

Le processus de professionnalisation des acteurs individuels et organisationnels de l'économie sociale (ES) est un phénomène abordé selon divers points de vue dans la littérature scientifique depuis de nombreuses années. On retrouve des définitions variées de cette notion, illustrant la polysémie du terme. La compréhension de ce processus est essentielle dans le contexte actuel, qui pousse un certain nombre d'entreprises d'économie sociale (EES) à se professionnaliser pour s'adapter à un environnement de plus en plus concurrentiel, aux exigences accrues de leur secteur d'activité ou aux attentes spécifiques de leurs partenaires. L'objet de cet article est, d'une part, de présenter les enjeux de la professionnalisation des EES et, d'autre part, d'offrir des pistes de réflexion quant aux solutions à envisager afin de réaliser une professionnalisation à la fois efficace et en adéquation avec les particularités des EES. 


\title{
LES ENJEUX
}

\section{DE LA PROFESSIONNALISATION DES ENTREPRISES D'ÉCONOMIE SOCIALE}

\author{
par Luc Audebrand et Myriam Michaud*
}

\begin{abstract}
Le processus de professionnalisation des acteurs individuels et organisationnels de l'économie sociale (ES) est un phénomène abordé selon divers points de vue dans la littérature scientifique depuis de participation à la rédaction des premières ébauches de cet article. nombreuses années. On retrouve des définitions variées de cette notion, illustrant la polysémie du terme. La compréhension de ce processus est essentielle dans le contexte actuel, qui pousse un certain nombre d'entreprises d'économie sociale (EES) à se professionnaliser pour s'adapter à un environnement de plus en plus concurrentiel, aux exigences accrues de leur secteur d'activité ou aux attentes spécifiques de leurs partenaires. L'objet de cet article est, d'une part, de présenter les enjeux de la professionnalisation des EES et, d'autre part, d'offrir des pistes de réflexion quant aux solutions à envisager afin de réaliser une professionnalisation à la fois efficace et en adéquation avec les particularités des EES.
\end{abstract}

* Université Laval, Québec. Les auteurs souhaitent remercier Gabrielle Magnin pour sa

\section{The issues of professionalization for social economy enterprises}

The professionalization process of individual actors and organisations in the social economy is a phenomenon that has been examined from various perspectives in the literature for many years. Different definitions of this notion can be found, illustrating the term's ambiguity. Understanding this process is essential in the current climate, which has been driving a number of social economy enterprises towards greater professionalization to adapt to an increasingly competitive environment, the growing demands of their business sector, and the specific requirements of their partners. The aims of this article are to present the issues of professionalization for social economy enterprises and suggest areas to be considered with regards to possible solutions for achieving a professionalization process that is both effective and appropriate for the particular features of social economy enterprises.

\section{Los desafíos de la profesionalización de las empresas de economía social}

El proceso de profesionalización de los actores individuales y organizativos de la economía social (ES) es un fenómeno abordado por diversas perspectivas en la literatura científica desde hace muchos anos. Se encuentran diversas definiciones de este concepto, ilustrando el carácter polisémico del término. La comprensión de este proceso es fundamental en el contexto actual, que anima algunas empresas de economía social (EES) a profesionalizarse para adaptarse a un entorno cada vez más competitivo, a las exigencias crecientes o las expectativas específicas de sus interlocutores. El tema de este artículo es, por un lado, plantear los desafíos de la profesionalización de las EES, y por otro lado ofrecer elementos de reflexión sobre las soluciones que se deben considerar para realizar una profesionalización eficaz y en adecuación con las particularidades de las EES. 
L'économie sociale (ES) a, selon Demoustier (2004), une capacité importante à créer de nouveaux emplois, voire de nouveaux métiers. Selon cette auteure, quatre défis devraient être relevés par l'ES: l'insertion en emploi de personnes provenant de groupes marginalisés, la création de nouveaux emplois par de nouvelles activités, l'amélioration de la qualité et de la productivité des services et, enfin, la lutte contre la pauvreté. Atteindre ces objectifs ambitieux mais réalistes passe cependant par une professionnalisation accrue des entreprises d'économie sociale (EES) et des individus qui les composent. Chanut-Guieu (2009, p. 15) estime que «l'obligation de résultats est [aujourd'hui] de mise dans tout type d'organisation et concourt à la professionnalisation des associations ». Cette obligation de performance, couplée à la complexification des tâches, justifierait alors la professionnalisation des EES.

La professionnalisation dans l'ES est un processus abordé selon divers points de vue dans la littérature scientifique. On retrouve des définitions variées pour décrire cette notion, illustrant la polysémie du terme (Cumming, 2008; Bureau, Suquet, 2009). La professionnalisation concerne plusieurs secteurs, et par-dessus tout celui de l'ES, encore largement constitué de bénévoles (Hwang, Powell, 2009). La compréhension de ce processus est donc essentielle dans le contexte actuel, qui pousse un certain nombre d'EES à se professionnaliser pour s'adapter aux changements de leur environnement, aux exigences de leur secteur d'activité ou aux attentes de leurs partenaires (Sanchis-Palacio, Campos-Climent, Mohedano-Suanes, 2013; Eng, Liu, Sekhon, 2012). Une telle évolution n'est pas sans conséquences pour ces entreprises qui sont caractérisées par la primauté de leur mission sociale (Hvenmark, 2013), mais doivent en même temps rencontrer des impératifs économiques.

D'une part, la professionnalisation peut référer à la constitution d'une profession, à travers la volonté collective des travailleurs d'être reconnus comme professionnels. Puissant (2011) relève deux cas possibles: la constitution d'une nouvelle profession (développement d'un savoir-faire, de règles reconnues et d'une déontologie) ou la reconnaissance officielle d'un métier préexistant. Quelle que soit la situation, la quête de légitimité et de reconnaissance sociale est l'objectif sous-jacent. Ainsi, selon Hugues (1996, cité dans Flahault et Dussuet, 2010, p. 36), la professionnalisation est le "processus de reconnaissance, par l'ensemble social, de l'exclusivité à exercer et de la légitimité à réguler l'activité dans un domaine déterminé». Cette perspective sociologique se retrouve dans une partie importante de la littérature sur le sujet (Flahault, Dussuet, Loiseau, 2009; George, 2013; Puissant, 2011).

D’autre part, la professionnalisation peut aussi représenter un processus de spécialisation et d'élévation des compétences (Bernardeau-Moreau, 2006). L'accent est alors mis sur le processus de formation en tant que tel, dans le but d'acquérir des compétences propres à une activité reconnue comme professionnelle. Dubruc et Badour (2011, p. 49) considèrent que la professionnalisation est avant tout une démarche pour " devenir un professionnel dans le cadre d'une formation co-construite en appui sur une analyse réflexive de sa pratique». Enfin, la professionnalisation des acteurs et des EES peut être étudiée non pas selon les formes qu'elle prend, mais d'après les conséquences qu'elle engendre. Cette notion désignerait alors un processus rapprochant les EES des entreprises du système dominant, par l'importation des pratiques 
et des idéologies attenantes. Jenkins (2005) décrit la professionnalisation comme un instrument clé du projet néolibéral, en tant qu'elle contribue à la cooptation, à la fusion et à la neutralisation des idéologies alternatives. Ce rapprochement entre la professionnalisation et l'idéologie néolibérale est aussi examiné dans les travaux de Broadbridge et Parsons $(2003 ; 2004)$, qui observent que le secteur caritatif devient ainsi plus orienté commercialement et semblable aux grandes entreprises.

A ce jour, il n'existe pas une compréhension du phénomène de professionnalisation qui soit spécifique à l'ES et qui serait uniformément partagée par les auteurs et les acteurs sur le terrain. Notre objectif est de combler cette lacune en présentant les résultats d'une recension des écrits sur ce phénomène. En première approximation, nous définissons la professionnalisation comme la stratégie d'évolution des activités traditionnelles de l'ES, marquées notamment par le bénévolat ou l'amateurisme, vers un statut dit professionnel, c'est-à-dire nécessitant des compétences spécifiques pour l'exercer. Face à la complexité de cette notion, il est important d'examiner les éléments de la littérature scientifique traitant des différentes motivations, stratégies et conséquences du processus de professionnalisation dans les EES, tant du point de vue organisationnel qu'individuel. Ces trois dimensions de la professionnalisation des EES feront l'objet des trois premières sections de cet article. La quatrième servira à dégager les stratégies les plus adaptées pour subir les impacts négatifs de ce processus.

\section{Les motivations qui sous-tendent la professionnalisation}

Les raisons menant des organisations ou des acteurs de l'ES à se professionnaliser dépendent du contexte dans lequel ils évoluent, mais aussi des objectifs que ceux-ci veulent atteindre par ce processus. On retrouve au moins quatre types d'incitatifs à la professionnalisation: pression externe, volonté, adaptation en interne à la recherche d'efficience et de qualité et désir de reconnaissance et de légitimité.

\section{Une réponse à une pression externe}

Un premier type d'incitatif, généralement abordé par la notion d'isomorphisme, regroupe les cas d'organisations qui se lancent dans un processus de professionnalisation à la suite d'une pression externe directe. Le concept d'isomorphisme défini par DiMaggio et Powell (1983) inclut trois mécanismes de changements organisationnels de nature isomorphique: coercitifs, normatifs ou mimétiques. Leiter (2013) démontre que les EES sont davantage portées à imiter les organisations évoluant dans le même secteur d'activité ou la même industrie, plutôt que les organisations ayant la même forme légale (entreprise privée, coopérative, association, etc.). Ainsi, la professionnalisation des entreprises capitalistes du même secteur d'activité exerce une pression isomorphique sur les EES. Le recours à des consultants déjà professionnalisés mène à l'adoption de pratiques reconnues par ces experts et confirme la présence d'un isomorphisme normatif entre les organisations d'un même secteur d'activités. 
La pression externe qui induit la professionnalisation dans les EES est fréquemment liée à des enjeux de financement. Sous l'angle de l'isomorphisme, une telle pression serait qualifiée de coercitive, en ce qu'elle provient d'une structure dont est dépendante l'organisation qui se professionnalise. Cette pression externe peut ainsi être exercée par les bailleurs de fonds, qui veulent s'assurer que l'organisation financée atteint des critères précis de performance et d'efficacité (Benjamin, 2008; Cumming, 2008). La relation de dépendance qui existe entre donateurs et bénéficiaires influence les pratiques adoptées par l'organisation, et par là même le processus global de professionnalisation. Or, dépendre financièrement de structures externes peut mener à modifier ses pratiques pour respecter leurs exigences (Verbruggen, Christiaens, Milis, 2011). La professionnalisation comme réponse à une pression externe se matérialise ainsi parfois par l'adoption d’outils inadaptés aux EES (Petrella, Richez-Battesti, 2010).

\section{Une recherche d'efficience et de qualité}

Si l'incitatif d'efficacité et de qualité est généralement en lien avec une pression externe, cette dernière n'est pas toujours aussi directe que dans les situations précédentes. L'organisation peut décider de se professionnaliser pour s'adapter à son environnement, sans nécessairement être soumise à une pression de la part de ses partenaires. La professionnalisation est, dans ce cas, avant tout liée à une vision du bénévolat comme étant incapable de fournir un service d'une efficience ou d'une qualité optimale (BernardeauMoreau, 2006; Hwang, Powell, 2009). Dans leur étude, Ganesh et McAllum (2012) distinguent le processus de professionnalisation du professionnalisme en lui-même, lequel réfère davantage à l'identité et aux pratiques communes d'une activité. La professionnalisation serait alors un moyen pour atteindre un certain niveau de qualité. Dès lors, se professionnaliser apparaît d'autant plus nécessaire si l'entreprise d'ES offre un service commercial et souhaite exceller dans ce domaine.

Petrella et Richez-Battesti (2010) présentent l'utilisation de démarches qualité par les EES comme une façon de s'adapter à leur environnement concurrentiel. L'adaptation à l'environnement est ainsi l'une des justifications majeures aux changements entrepris par les EES qui se professionnalisent pour prouver la qualité de leurs services. Pour sa part, Pattieu (2010) examine le cas d'une association dans le domaine du tourisme social, dont le passage progressif en société commerciale, par l'application des normes de gestion issues du secteur lucratif, était vu comme la solution adéquate aux problèmes de qualité auxquels elle faisait face. Toutefois, il s'est révélé que le principal problème de cette organisation ne relevait pas de la forme juridique associative, mais du modèle de syndicalisme français dont elle avait hérité. L'auteur en conclut que la professionnalisation n'est pas forcément une réponse adaptée aux problèmes rencontrés.

Pour Sanchis-Palacio, Campos-Climent et Mohedano-Suanes (2013), l'utilisation d'outils de gestion stratégique constitue un facteur d'amélioration de l'efficacité sociale des EES, au sens de leur degré de réussite dans la réalisation de leurs objectifs sociaux, notamment en matière de création d'emploi. Ainsi, ces auteurs affirment que plus une organisation 
aura recours à des outils de gestion stratégique, plus elle sera socialement efficace et considérée comme professionnalisée. Cela justifierait la volonté de professionnalisation exprimée par certaines EES qui cherchent à améliorer leur performance sociale.

\section{Un processus d'adaptation volontaire}

Si l'on observe fréquemment la présence d'une pression externe directe ou indirecte menant à se professionnaliser, les entreprises et les acteurs de l'ES peuvent être eux-mêmes à la source de cette démarche. Par exemple, Alonso et Maciel (2010) examinent le cas de la professionnalisation volontaire des mouvements de défense environnementale au Brésil. La stratégie de professionnalisation visait ici à passer d'une approche de protestation à une approche de négociation, moins agressive. Il apparaît que les associations étudiées, nouvellement professionnalisées, sont devenues plus efficaces et influentes que les partis verts brésiliens. Précisons que, dans cet exemple, si l'adaptation est à l'origine volontaire, le processus de professionnalisation a néanmoins comme ultime objectif de mieux faire entendre leurs revendications. De même, Lundström (2001) constate parmi certaines organisations de protection de l'enfance l'émergence d'un besoin d'acquérir une expertise dans leur domaine, motivé par les exigences croissantes du secteur. Pour pouvoir continuer à réaliser leur mission convenablement, les organisations désirent se spécialiser davantage, en formant leurs bénévoles.

Ainsi, que la professionnalisation soit volontairement initiée ou encouragée par des pressions externes, ce processus s'inscrit dans une démarche d'adaptation et de réactivité face à l'environnement. Cela montre aussi un mouvement volontaire de professionnalisation incarné par des acteurs qui recherchent une place plus influente dans leur domaine d'activité.

\section{Une quête de reconnaissance et de légitimité}

La professionnalisation survient aussi au sein de groupes de travailleurs souhaitant obtenir une reconnaissance officielle de leurs compétences et la légitimité de leur contribution à la société. Parmi les cas observés, on remarque que la professionnalisation s'accompagne généralement d'un mouvement collectif. Les groupes de travailleurs vont s'organiser et mutualiser leurs forces pour obtenir la reconnaissance de leur activité. George (2013) détaille ainsi les circonstances par lesquelles les travailleurs d'une occupation donnée (ici, les " coachs de vie») tentent de la transformer en une profession pour que les travailleurs soient eux-mêmes considérés comme des professionnels. Cette façon de désigner le processus met l'accent sur deux composantes: la volonté collective relative à la professionnalisation de l'activité en tant que telle et la volonté individuelle relative à l'obtention d'un certain niveau de professionnalisme. George conclut que la simple cohabitation de diverses stratégies individuelles n'aboutit pas nécessairement à une professionnalisation de l'activité aussi réussie qu'une stratégie portée par un mouvement collectif de quête de reconnaissance.

Bureau et Suquet (2009) affirment que le phénomène de professionnalisation touche particulièrement les occupations en quête de légitimité. 
Cette notion est aussi primordiale pour George (2013), qui ajoute que les travailleurs veulent non seulement obtenir la reconnaissance sociale de l'utilité de leur occupation, mais aussi la légitimité et la crédibilité qui y sont associées. Puissant (2011) expose le rôle important des associations d'aide à domicile dans la professionnalisation des travailleurs et de l'activité en elle-même. Le cadre collectif de travail fait des associations des employeurs collectifs "en mesure de mettre en œuvre une dynamique de professionnalisation»(Puissant, 2011, p. 47). Là encore, la reconnaissance obtenue semble intimement liée à la force du mouvement collectif de professionnalisation.

\section{Les différentes voies de professionnalisation}

La diversité du concept de professionnalisation se retrouve aussi dans les moyens qui sont employés par les EES pour mener à bien ce processus d’adaptation. La stratégie suivie et les outils utilisés s’inspirent plus fréquemment du modèle économique dominant que de l'ES (Battilani, Zamagni, 2012) et se distinguent en fonction de leur potentiel transformateur.

\section{La formation du personnel}

Cette forme de professionnalisation est relativement répandue au sein des EES (Chanut-Guieu, 2009; Dubruc, Badour, 2011). Elle présente l'avantage de mettre à contribution les capacités des travailleurs d'origine, qui développent leurs compétences, se spécialisent et acquièrent une expertise dans leur domaine. C'est ce processus qui est illustré par les cas des bénévoles d'organisations de protection de l'enfance (Lunderström, 2011) et de l'activisme environnemental au Brésil (Alonso, Maciel, 2010). Dans cette dernière situation, le processus s'est effectué sur deux niveaux: la professionnalisation des organisations en tant que telles et la spécialisation des individus. Par exemple, l'apparition d'une spécialisation technique des activistes brésiliens, combinée à la formalisation des associations, a causé certaines modifications aux principes qui caractérisaient à l'origine ce mouvement activiste, transformant les protestations de rue en négociations calculées.

La professionnalisation comme processus d'évolution des ressources humaines en interne s'accompagne très fréquemment d'un passage du bénévolat au salariat (Chanut-Guieu, 2009). Un tel processus donne lieu à une cohabitation, temporaire ou permanente, entre ces deux formes de travail au sein de l'organisation qui se professionnalise. Cette évolution est parfois présentée comme indispensable, face aux enjeux d'efficacité, de performance et de compétences nécessaires pour réaliser les tâches à accomplir. Toutefois, le passage du bénévolat au salariat peut mettre à mal les valeurs associatives, recherchées par les membres et la communauté (Falcoz, Walter, 2007).

De même, on observe une tendance à la professionnalisation parmi les dirigeants d'EES. Dubruc et Badour (2011) exposent le cas de la professionnalisation des directeurs associatifs par la formation continue. Ce phénomène s'inscrit dans une démarche d’isomorphisme mimétique, 
car les associations étudiées ont eu recours à des solutions déjà reconnues dans leur secteur d'activité. Toutefois, les dirigeants concernés veulent conserver leur identité, tout en s'adaptant aux contraintes de leur environnement. Ainsi, si cette forme de professionnalisation a un potentiel transformateur, les changements induits peuvent s'adapter aux spécificités de l'organisation. C'est tout l'intérêt de cette stratégie de professionnalisation. Celle-ci peut se révéler moins brutale que l’importation directe d’idéologies, de pratiques ou de professionnels, qui appartiennent généralement au secteur de l'économie privée et auront ainsi un impact plus fort sur l'entreprise d'ES (Hvenmark, 2013).

\section{Le recrutement de personnel}

Cette forme de professionnalisation résulte de l'influence des professionnels extérieurs auxquels les EES peuvent avoir recours (Hvenmark, 2013; Broadbridge, Parsons, 2004). La tendance actuelle semble être d'effectuer le recrutement de ces nouvelles ressources humaines dans le secteur privé (Parente, 2012). Cela peut poser problème, car une telle stratégie ne prend pas nécessairement en compte les valeurs de l'ES.

Parente (2012) a ainsi étudié la transition professionnelle d'EES au Portugal. Parmi les nouveaux employés, la majorité occupait auparavant un poste dans le secteur public ou privé, mais jamais dans le tiers secteur. Parente considère que cela illustre le sens des transferts de compétences, s'effectuant plus fréquemment du privé vers l'ES, et plus rarement entre les EES. Dans son exemple, des problèmes sont apparus entre les nouveaux salariés, diplômés, et leurs supérieurs hiérarchiques, souvent bénévoles, mais avec une grande expérience "terrain ». L'auteure observe en effet que les cadres sont porteurs des valeurs de l'ES, tandis que les salariés diplômés auraient pour vocation d'assurer un niveau d'efficacité, grâce à leurs compétences techniques.

Cette tension possible entre personnel « qualifié » et personnel « dévoué » est également relevée par Hvenmark (2013), pour qui le personnel qualifié serait plus enclin à accepter l'importation de pratiques de l'économie capitaliste. Ainsi, la professionnalisation par l'emploi de personnel déjà formé peut avoir une influence sur les valeurs d'origine de l'entreprise d'ES.

\section{L'importation de pratiques de l'idéologie dominante}

Les EES ont souvent tendance à s'inspirer des entreprises capitalistes pour se professionnaliser (Ughetto, Combes, 2010). Al'instar de l'emploi de personnel déjà formé, le choix d'une stratégie de managérialisation (Hvenmark, 2013; Battilani, Zamagni, 2012) ou de commercialisation (Dart, 2004; Eikenberry, 2009) de l'activité de l'entreprise d'ES aura des conséquences sur les principes et les valeurs de l'organisation, ainsi que sur la façon d'aborder sa mission sociale (Broadbridge, Parsons, 2003).

La notion de commercialisation est récurrente dans la littérature scientifique (Åberg, 2013; Eikenberry, 2009; Frumkin, Andre-Clark, 2000). Ce processus a lieu lorsqu'une entreprise d'ES développe une motivation pour le profit (Dart, 2004). Concrètement, l'organisation concernée par cette évolution utilisera des pratiques et des outils initialement destinés aux entreprises 
capitalistes, dans le but d'accomplir sa mission sociale. Deux études de Broadbridge et Parsons $(2003$; 2004) abordent la commercialisation du secteur caritatif au Royaume-Uni, montrant comment l'embauche de cadres professionnels provenant du secteur privé peut introduire une culture d'entreprise contradictoire avec les objectifs caritatifs à l'origine de l'association. La professionnalisation désigne alors ces changements qui mènent les EES à être plus orientées vers le profit, induisant de nouvelles pratiques générant de l'uniformité dans le secteur caritatif.

La managérialisation constitue une autre forme de professionnalisation des EES, qui consiste à mobiliser des techniques du management moderne (Battilani, Zamagni, 2012) ou encore à adopter des normes et des valeurs du secteur privé (Carvalho, 2012). Elle peut également être présentée comme la dépendance croissante à l'idéologie et aux pratiques importées du management corporatif (Hvenmark, 2013). Quelle que soit la définition retenue, l'influence de cette idéologie, étrangère aux EES car provenant de l'économie dominante, est une constante de la notion de managérialisation. Le cas traité par Hvenmark (2013) est celui de l'adoption d'un outil du management stratégique, le tableau de bord prospectif (Balanced Scorecard), par des EES. Cette pratique a un impact au niveau idéologique, en prônant le recours à des solutions techniques tout en induisant la conviction selon laquelle le niveau de formation professionnelle détermine les compétences des individus. Selon l'auteur, cette idéologie managériale a pour ultime conséquence de présenter les entreprises capitalistes comme les exemples à suivre et, par cela, d'orienter les EES vers le marché dominant. Cette démarche les éloigne de leur mission sociale autant que de leurs valeurs d'origine, qui constituent pourtant des avantages distinctifs. De surcroît, l'adoption du tableau de bord prospectif s'est révélée inefficace dans le cas observé.

Ces deux dernières formes de professionnalisation peuvent par conséquent avoir des effets plus ou moins positifs pour l'entreprise d'ES qui les utilise. Toutefois, certains auteurs considèrent que la commercialisation offre la possibilité aux EES de se professionnaliser tout en préservant leur autonomie et leur mission sociale, puisqu'elle réduit leur dépendance financière (Eng, Liu, Sekhon, 2012). Mitchell (2014) interprète également la commercialisation comme une stratégie ayant pour but de maintenir l'autonomie financière et organisationnelle des structures par rapport aux pouvoirs publics et autres apporteurs de fonds. Ces points de vue, à contre-courant de l'analyse qui vient d'être faite des stratégies, n'en sont pas moins importants.

\section{Les écueils de la professionnalisation des EES}

Lorsque la stratégie de professionnalisation n'est pas adaptée aux EES, ce processus peut se révéler dommageable. Dans le cas présent, l'« adaptation » consiste à prendre en compte les spécificités des EES (prépondérance de la mission sociale sur la mission économique, hybridation des ressources - notamment le recours au bénévolat -, autonomie et indépendance par rapport à l'Etat, structure démocratique, etc.) lors de l'initiation du processus de professionnalisation. Cela permettrait d'éviter les principaux écueils rencontrés par les EES qui se professionnalisent. 


\section{La perte du caractère distinctif}

L'idée selon laquelle la professionnalisation mènerait à une homogénéisation des EES est récurrente dans la littérature. Ce point apparaît comme négatif, puisque l'une des forces des EES réside précisément dans leur grande diversité (Leiter, 2013). Eikenberry (2009) va jusqu'à affirmer que si l'adoption des outils issus du modèle économique dominant est néfaste, l'adoption d'un modèle commun à l'ensemble des EES le serait tout autant, puisqu'elle mettrait en péril cette diversité. Ce risque de banalisation peut en outre nuire à un autre avantage distinctif des EES, à savoir la force mobilisatrice de leurs valeurs fondatrices, exprimées directement dans leur mission sociale (Frumkin, Andre-Clark, 2000). Ce phénomène d'homogénéisation des EES causé par le mouvement de professionnalisation se rencontre dans plusieurs secteurs. Ainsi, selon BernardeauMoreau (2006, p. 150), le secteur associatif «devient de plus en plus apparenté au monde de l'entreprise et du travail », les valeurs militantes étant délaissées au profit des nouvelles exigences managériales du milieu. De même, pour Petrella et Richez-Battesti (2010), l'utilisation d'outils de mesure de la qualité, issus du secteur privé, risque de mener à une perte d’identité pour les EES. Broadbridge et Parsons (2003) évoquent quant à elles l'apparition d'une certaine uniformité dans le secteur caritatif, sous l'effet de la professionnalisation. Cela s'observe même au niveau individuel: ainsi, la fonction de dirigeant d'entreprise d'ES, en se professionnalisant, tend à être banalisée par l'uniformité des formations proposées, qui génère des profils similaires (Lazuech, 2006).

\section{La « marketization » et les conflits de valeurs}

L’image de l'infiltration de l'idéologie de marché jusque dans les sphères de l'ES, à travers les méthodes qu'elle emploie pour se professionnaliser, est également exprimée par le terme "marketization». Cette notion illustre la domination exercée par l'économie capitaliste; domination qui s'incarne dans les pratiques à suivre et les façons de penser prescrites par ce marché omniprésent (Eikenberry, 2009). Cela risque d'entraîner la perte des valeurs initiales de l'entreprise sociale, via l'utilisation de techniques et d'outils managériaux issus des entreprises capitalistes (Broadbridge, Parsons, 2004), qui ne sont pas nécessairement adaptés à la complexité des EES. Celles-ci doivent en effet remplir une mission sociale tout en répondant à des exigences économiques. La perte des valeurs de l'entreprise d'ES pourrait ainsi finalement l'éloigner de sa mission initiale à cause de l'influence des pratiques importées du secteur privé (Valentinov, 2012).

Comme mentionné précédemment, l'un des effets de la commercialisation en tant que stratégie de professionnalisation est de développer une motivation nouvelle pour le profit (Dart, 2004). Broadbridge et Parsons (2003) mettent également en évidence l'un des effets de la commercialisation pour les EES, qui crée un conflit de valeurs entre les cadres dirigeants, plus axés sur les résultats commerciaux, et les cadres de terrain, plus soucieux des principes caritatifs. Dans ce cas, c'est la mission même de l'entreprise qui est en jeu. Selon Åberg (2013), la professionnalisation et la commercialisation à l'œuvre parmi certaines organisations de la société civile se font aux dépens de la tradition organisationnelle démocratique et des valeurs initiales, compromettant leur légitimité d’un point de vue sociétal. 


\section{Pistes de solution : la professionnalisation dans le respect des valeurs initiales}

Les écueils que connaissent les structures professionnalisées de l'ES sont nombreux: l'intérêt d'adapter les stratégies de professionnalisation aux spécificités des EES en est d'autant plus évident. Car, dans les faits, il serait possible d'utiliser des outils de gestion provenant de l'économie classique tout en conservant sa mission sociale (Malo, Audebrand, Camus, 2008). Malgré les risques idéologiques qui résultent de l'import de pratiques et d'outils du secteur privé, les EES doivent en effet plus que jamais s'adapter à leur environnement, répondre aux demandes des partenaires financiers, accompagner les aspirations des travailleurs et des bénévoles, s'adapter aux exigences de leur secteur d'activité et gagner la confiance de leurs membres ou de leurs clients. Pour cela, elles doivent devenir de plus en plus efficientes dans les différents domaines stratégiques reliés à leur activité principale. L'enjeu est de ne pas perdre en chemin ses valeurs initiales. Ce risque pourrait constituer un frein à la professionnalisation, car les individus concernés par le processus peuvent craindre de perdre leurs valeurs au profit de celles qui sont véhiculées par les outils de l'économie classique (Van Der Yeught, Bergery, 2012).

Certains auteurs donnent des conseils généraux pour mener à bien le processus de professionnalisation. Du point de vue organisationnel, Frolic (2012) propose d'impliquer les parties prenantes, d'investir dans le renforcement des relations pour établir la confiance, de faire un plan d'action précis et, enfin, de se doter d'une méthode de délibération qui permette de consulter toutes les personnes concernées par le processus. Du point de vue de la professionnalisation des individus, Chanut-Guieu (2009) conseille de suivre trois principes: formation, information et transition progressive. Selon cette auteure, la stratégie de professionnalisation adoptée doit impérativement répondre aux spécificités des EES auxquelles elle s’applique.

\section{Le développement de compétences spécifiques}

Faire fonctionner une entreprise d'ES nécessite des compétences spécifiques. Ces structures se caractérisent effectivement par une dualité entre leur finalité sociale et les impératifs que toute organisation économique rencontre. La difficulté est donc de réussir à concilier ces deux pôles de façon équilibrée. D'où la pertinence de penser qu'une entreprise d'ES ne peut pas adopter aveuglément des pratiques destinées initialement aux entreprises capitalistes. Il faut les adapter ou développer de nouveaux outils spécifiques pour les EES (Sanchis-Palacio, Campos-Climent, 2007). Par ailleurs, Valéau (2013, p. 91) suggère que « rien ne pourra se faire sans des directeurs et des DRH [directeurs des ressources humaines] formés aux spécificités du mouvement associatif ». Cet auteur insiste sur l'importance de former les gestionnaires aux particularités des EES, au regard de l'influence que ceux-ci peuvent avoir sur le déroulement du processus de professionnalisation.

Il semble ainsi également nécessaire d'envisager la professionnalisation sous l'angle de l'adaptation ou de la formation des acteurs aux impératifs de l'ES elle-même. Cuénoud, Moreau et Mertens (2013) proposent une liste des compétences spécifiques requises pour diriger une entreprise d'ES, parmi lesquelles 
"soutenir les objectifs multiples des entreprises sociales» ou encore "gérer le personnel rémunéréou bénévole». Par ailleurs, en réponse à cette cohabitation souvent difficile des salariés et des bénévoles au sein d'une organisation, Lazuech (2006, p. 72) suggère le rôle de cadre militant, une «figure sociale nouvelle qui seraitfaçonnée entièrement parcette double appartenance ». Celuici ne serait ni un cadre devenu militant, ni l'inverse, mais bien une nouvelle figure à part entière. L'intérêt serait d'avoir un modèle de dirigeant qui soit adapté aux valeurs de l'ES, tout en maintenant un haut niveau de professionnalisme dans ses fonctions de gestion. La reconnaissance d'une telle figure managériale serait importante, pour ses effets en termes de légitimité, mais aussi pour la possibilité de concilier à la fois les intérêts des bénévoles et ceux des salariés au sein d'une entreprise d'ES qui se professionnalise.

\section{Regroupements : réseaux et fédérations}

Un frein à la professionnalisation identifié par Puissant (2011) est le manque de collaboration entre les associations. L'émiettement en petites structures serait ainsi un obstacle à la réussite d'un mouvement de professionnalisation des EES. Si le cas étudié dans l'article de Puissant est celui des aides à domicile, il semble que l'observation soit généralisable à d'autres situations: les fédérations peuvent soutenir le processus de professionnalisation de façon intéressante, et contrer, par cela, ce problème d'émiettement. Dubruc et Badour (2011, p. 60) indiquent que «le poids du réseau nationalet sa façon de mobiliser ses associations adhérentes sont deux éléments importants dans la réussite d'un tel dispositif [de professionnalisation] », notamment au moyen d'un partenariat entre le réseau national d'associations étudié et un institut de formation.

Harter et Krone (2001) examinent quant à eux la contribution d'une organisation de soutien coopératif dont l'objectif est d'aider les coopératives qui en sont membres à évoluer en fonction de leur environnement, tout en conservant leur idéologie originelle. Si la notion de professionnalisation n'est pas directement abordée par les auteurs, l'article permet de comprendre et de mesurer la capacité de soutien d'une organisation intermédiaire. L'adaptation à l'environnement est ici présentée comme nécessaire, mais l'article montre avant tout la possibilité de mener ce changement sans s'éloigner des valeurs initiales de l'organisation. Ce résultat pourrait être transposé au processus de professionnalisation en tant que tel: les regroupements d'organisations sous forme de fédérations, de réseaux ou autour d'une structure intermédiaire semblent offrir de nombreux avantages pour mener à bien un mouvement de professionnalisation. Ces différentes formes de regroupement permettent en effet de mutualiser les moyens dans le but d'atteindre un objectif commun. Si les auteurs se rejoignent sur l'efficacité de la fonction de soutien des regroupements, il n'est pas encore possible de déterminer si ces structures intermédiaires favorisent systématiquementl'adoption d'outils de professionnalisation destinés avant tout aux EES. Néanmoins, Battilani et Zamagni (2012), dans leur analyse de l'histoire de la transformation des coopératives italiennes, observent que si les umbrella organizations favorisaient autrefois un processus de managérialisation qui n'était pas toujours en accord avec l'identité de la coopérative, ces mêmes organisations jouent aujourd'hui un rôle de taille dans la préservation des valeurs des coopératives adhérentes. 


\section{Conclusion}

Afin de relever les ambitieux défis qu'elle s'est lancés - insertion de personnes marginalisées sur le marché du travail, création de nouveaux emplois par de nouvelles activités, amélioration de la qualité et de la productivité des services, lutte contre la pauvreté (Demoustier, 2004) -, il apparaît impératif que l'ES poursuive son virage vers une plus grande professionnalisation. Celui-ci doit cependant se faire dans le respect des valeurs fondamentales de l'ES et de la mission sociale de ces entreprises. Parmi les solutions pour une professionnalisation réussie, il est suggéré de développer des compétences de cadre militant (Lazuech, 2006), de travailler en réseau ou au sein de regroupements (Puissant, 2011) et de créer des outils techniques et conceptuels adaptés (Malo, Audebrand, Camus, 2008). La multiplication de tels outils faciliterait grandement le parcours de professionnalisation des EES, leur permettant de s'adapter à leur environnement tout en conservant leurs valeurs d'origine (Sanchis-Palacio, Campos-Climent, 2007).

A ce stade de la réflexion sur la professionnalisation, des recherches empiriques sont nécessaires pour mieux appréhender les besoins de formation des EES. Notamment, le thème des transferts de connaissances et de compétences qui sont envisageables entre les EES elles-mêmes constitue un terrain de recherche important et stimulant. Par ailleurs, une meilleure compréhension des facteurs de réussite des processus de professionnalisation des EES met au jour les besoins spécifiques de ces organisations. Enfin, une réflexion renouvelée, ancrée dans le contexte socioéconomique actuel, sur la ou les distinctions spécifiques du secteur de l'ES serait nécessaire afin de mieux cerner ce qui doit être soigneusement préservé dans tout processus de changement chez les EES, incluant celui de la professionnalisation. 


\section{BiBLIOGRAPHIE}

Åberg P., 2013, "Managing expectations, demands and myths: Swedish study associations caught between civil society, the State and the market », Voluntas, n² 24(3), p. 537-558.

Alonso A. Maciel D., 2010, « From protest to professionalization: Brazilian environmental activism after Rio-92 ", Journal of Environment \& Development, $\mathrm{n}^{\circ}$ 19(3), p. 300-317.

Battilani P., Zamagni V., 2012, « The managerial transformation of Italian co-operative enterprises 1946-2010 », Business History, n 54(6), p. 964-985.

Benjamin L. M., 2008, " Account space: How accountability requirements shape nonprofit practice ", Nonprofit and Voluntary Sector Quarterly, n³7(2), p. 201-223.

Bernardeau-Moreau D., 2006, Bénévolat et fédération sportive, de l'association à l'entreprise, de l'intérêt général à l'utilité sociale: la reconfiguration de l'action publique entre Etat, associations et participation citoyenne, p.147162, Paris, éd. L'Harmattan.

Bureau S., Suquet J., 2009, «A professionalization framework to understand the structuring of work ", European Management Journal, $n^{\circ} 27(6)$, p. 467-475.

Broadbridge A., Parsons L., 2003, «Still serving the community? The professionalisation of the UK charity retail sector", International Journal of Retail \& Distribution Management, $n^{\circ} 31(8)$, p. 418-427.

Broadbridge A., Parsons E., 2004, « Managing change in nonprofit organizations: Insights from the UK charity retail sector ", Voluntas, $\mathrm{n}^{\circ}$ 15(3), p. 227-242.

Carvalho T., 2012, "Managerialism and professional strategies: A case from nurses in Portugal ", Journal of Health Organization and Management, $\mathrm{n}^{\circ}$ 26(4), p. 524-541.

Chanut-Guieu C., 2009, « La professionnalisation de la fonction de bénévole: quand l'Etat impulse le changement », Management \& Avenir, $\mathrm{n}^{\circ} 27, \mathrm{p} .13-30$.

Cuénoud T., Moreau C., Mertens S., 2013, « Les spécificités managériales dans les entreprises sociales: une démarche européenne par la gestion des compétences ", Recma, n³29, p. 80-91.

Cumming D., 2008, « French NGOs in the global era: Professionalization "without borders"? ", Voluntas, $\mathrm{n}^{\circ}$ 19(4), p. 372-394.

Dart R., 2004, "Being "business-like" in a nonprofit organization: A grounded and inductive typology ", Nonprofit and Voluntary Sector Quarterly, n³3(2), p. 290-310.

Demoustier D., 2004, L'économie sociale et solidaire face aux mutations du travail: l'expérience française, université du Québec en Outaouais, chaire de recherche en développement des collectivités (CRDC) du Canada.

DiMaggio P. J., Powell W. W., 1983, « The iron cage revisited: Institutional isomorphism and collective rationality in organizational fields ", American Sociological Review, $n^{\circ}$ 48(2), p. 147-160. Dubruc N., Badour M., 2011, « Professionnalisation des directeurs associatifs par la formation continue en alternance: le cas d'un réseau d'associations familiales ", Recma, n 322, p. 47-61. Eikenberry A. M., 2009, « Refusing the market: A democratic discourse for voluntary and nonprofit organizations ", Nonprofit and Voluntary Sector Quarterly, n 38(4), p. 582-596.

Eng T-Y., Liu C.-Y. G., Sekhon Y. K., 2012, « The role of relationally embedded network ties in resource acquisition of British nonprofit organizations ", Nonprofit and Voluntary Sector Quarterly, n 41(6), p. 1092-1115.

Falcoz M., Walter E., 2007, « Travailler dans un monde de bénévoles: contraintes et limites de la professionnalisation dans les clubs sportifs ", Recma, n 306, p. 78-91.

Flahault E., Loiseau D., Dussuet A., 2009, "Quelle gestion des ressources humaines dans l'économie sociale? Entre bénévolat et professionnalisation: la place du travail dans les associations », Eso Travaux et Documents, n² 28, p. 67-75. Flahault E., Dussuet A., 2010, «Entre professionnalisation et salarisation, quelle reconnaissance du travail dans le monde associatif?", Formation emploi, $\mathrm{n}^{\circ} 111$, p. 35-50. 
Frolic A., 2012, «Grassroots origins, national engagement: Exploring the professionalization of practicing healthcare ethicists in Canada", Healthcare Ethics Committee Forum: An Interprofessional Journal on Healthcare Institutions Ethical and Legal Issues, $\mathrm{n}^{\circ} 24(3)$, p. 153-164.

Frumkin P., Andre-Clark A., 2000, «When missions, markets, and politics collide: Values and strategy in the nonprofit human services ", Nonprofit and Voluntary Sector Quarterly, $\mathrm{n}^{\circ} 29$, p. 141-163.

Ganesh S., McAllum K., 2012, "Volunteering and professionalization: Trends in tension? », Management Communication Quarterly, $\mathrm{n}^{\circ} 26(1)$, p. 152-158.

George M., 2013, « Seeking legitimacy: The professionalization of life coaching », Sociological Inquiry, $\mathrm{n}^{\circ}$ 83(2), p. 179-208.

Harter L., Krone K., 2001, « The boundaryspanning role of a cooperative support organization: Managing the paradox of stability and change in non-traditional organizations ", Journal of Applied Communication Research, $n^{\circ}$ 29(3), p. 248-277.

Hvenmark J., 2013, «Business as usual? On managerialization and the adoption of the balanced scorecard in a democratically governed civil society organization ", Administrative Theory \& Praxis, $\mathrm{n}^{\circ} 35(2)$, p. 223-247.

Hwang H., Powell W. W., 2009, « The rationalization of charity: The influences of professionalism in the nonprofit sector", Administrative Science Quarterly, n 54(2), p. 268-298.

Jenkins K., 2005, « No way out? Incorporating and restructuring the voluntary sector within spaces of neoliberalism », Antipode, $\mathrm{n}^{\circ} 37(3)$, p. 613-618.

Lazuech G., 2006, « Les cadres de l'économie sociale et solidaire: un nouvel entreprenariat? ", Formation emploi, n ${ }^{\circ}$ 95, p. 59-74.

Leiter J., 2013, « An industry fields approach to isomorphism involving Australian nonprofit organizations », Voluntas, n²4(4), p. 1037-1070.
Lundström T., 2001, " Child protection, voluntary organizations, and the public sector in Sweden ", Voluntas, n²12(4), p. 355-372.

Malo M.-C., Audebrand L. K. Camus A., 2008, "Gestion stratégique des organisations du commerce équitable», Gestion, n 33(1), p. 85-94. Mitchell G. E., 2014, «Strategic responses to resource dependence among transnational NGOs registered in the United States ", Voluntas, $n^{\circ}$ 25(1), p. 67-91.

Parente C., 2012, "Qualified employment in the third sector in Portugal », Voluntas, $\mathrm{n}^{\circ} 23(1)$, p. 257-276.

Pattieu S., 2010, « Une association de tourisme social face à la professionnalisation du secteur: tourisme et travail ", Recma, n 89(318), p. 90-103.

Petrella F., Richez-Battesti N., 2010, « Régulation de la qualité dans les services à la personne en France: l'économie sociale et solidaire entre innovation et isomorphisme? ", Management \& Avenir, $n^{\circ} 35$, p. 273-292.

Puissant E., 2011, "Le rôle ambivalent des associations d'aide à domicile dans la professionnalisation des emplois et des salariées ", Formation emploi, $\mathrm{n}^{\circ} 115$, p. 37-50.

Sanchis-Palacio J., Campos-Climent V., 2007, "Strategic management in the social economy: Using strategic analysis tools in cooperatives », Ciriec-España, n 59, p. 237-258.

Sanchis-Palacio J., Campos-Climent V., Mohedano-Suanes A., 2013, " Management in social enterprises: The influence of the use of strategic tools in business performance ", International Entrepreneurship and Management Journal, n 9(4), p. 541-555.

Ughetto P., Combes M.-C., 2010, « Entre les valeurs associatives et la professionnalisation: le travail, un chaînon manquant?", Socio-logos: revue de l'association française de sociologie, $\mathrm{n}^{\circ} 5$. Valentinov V., 2012, " Toward a critical systems perspective on the nonprofit sector", Systemic Practice and Action Research, $\mathrm{n}^{\circ} 25(4)$, p. 355-364. 
Valéau P., 2013, « La fonction RH dans les associations: les valeurs militantes à l'épreuve de la professionnalisation ", Recma, n 328, p. 76-94. Van Der Yeught C., Bergery L., 2012, «Quelles compétences de l'entrepreneuriat social et solidaire transférer vers la petite entreprise responsable? », Revue internationale PME. : économie et gestion de la petite et moyenne entreprise, $\mathrm{n}^{\circ}$ 25(3-4), p. 155-194.

Verbruggen S., Christiaens J., Milis K., 2011, «Can resource dependence and coercive isomorphism explain nonprofit organizations' compliance with reporting standards?», Nonprofitand Voluntary Sector Quarterly, n 40(1), p. 5-32.

A Retrouver dans LeS ARCHives de LA « ReCMA » (RECMA.ORg/ARTICLES)

Valéau P., " La fonction RH dans les associations: les valeurs militantes à l'épreuve de la professionnalisation ", Recma, $\mathrm{n}^{\circ} 328,2013$.

Pattieu S., « Une association de tourisme social face à la professionnalisation du secteur: tourisme et travail », Recma, $\mathrm{n}^{\circ}$ 318, 2010.

Falcoz M., Walter E., «Travailler dans un monde de bénévoles: contraintes et limites de la professionnalisation dans les clubs sportifs », Recma, n 306, 2007.

Clergeau C., Dussuet A., "Les entreprises sociales en quête de professionnalisation, l'exercice difficile des associations d'aide à domicile aux personnes âgées », Recma, n 294 , 2004.

Delattre M., «Professionnalisme et bénévolat: alibi de l'amateurisme ou crise des discours?», Recma, n²83, 2002. 